\title{
Parallel hybrid electric propulsion architecture for single aisle aircraft - powertrain investigation
}

\author{
Jos Vankan ${ }^{1, *}$, Wim Lammen ${ }^{1}$ \\ ${ }^{1}$ Netherlands Aerospace Centre NLR, PO Box 90502, 1006 BM Amsterdam, jos.vankan@nlr.nl
}

\begin{abstract}
This paper presents an investigation of the fuel- and energysaving potential through the introduction of several hybrid electric propulsion (HEP) and more electric aircraft (MEA) systems on single aisle aircraft. More specifically, for an A320NEO the following main electric systems are considered: electric motors, batteries and power electronics for parallel HEP, electric components for replacement of the main pneumatic and hydraulic non-propulsive systems like environmental control system and actuators, and electric power transport and supply. The power sizing of the electric components, as well as their mass effects on overall aircraft mission performance are evaluated by system modelling of the aircraft, turbofan and the considered electric components. It is found for the considered aircraft and missions that the fuel saving potential of parallel HEP systems alone is very limited or absent. Typically the combination of HEP and MEA technologies shows potential for improved energy efficiency due to synergies of the involved systems and their operation.
\end{abstract}

\section{Introduction}

In response to the ongoing strong growth in air traffic (e.g. [1]) and its impact on the natural environment, ambitious targets and roadmaps for future aviation have been defined (e.g. [2], [3]). One of the key components to achieve the necessary reduction of fossil fuel consumption and global air traffic emissions is the further advancement of airframe and propulsion innovations and the related technology developments. Hybrid electric propulsion (HEP) has been identified as one of the potential solution areas [4]. HEP systems were first introduced on a large scale in the automotive sector and are now making their way to the aviation industry. These HEP systems attempt to reduce fuel consumption and emissions of traditional combustion engines through hybridisation via electrical energy sources. Another trend in the aviation industry is the electrification of aircraft subsystem architectures. Such so-called "more electric aircraft" (MEA), as for example the Airbus A350 or the Boeing 787, feature advanced electrically powered subsystems instead of the conventional hydraulic and pneumatic counterparts for non-propulsive on-board functions.

With the current state of technology, full electrification of propulsion for large civil aircraft and medium haul or long haul flight is not realistic. This is because such flights require very high levels of power and energy. With the current relatively low values of 
specific power and specific energy of state-of-the-art electrical systems, this would lead to very high mass of the electric propulsion and energy storage system. Therefore it is expected for the next decades that gas turbines will remain to play a crucial role in propulsion of large aircraft. But it is also expected that the combination of gas turbines with electric motors in HEP systems has potential to reduce fuel consumption and gas and noise emissions of large aircraft.

A variety of system architectures can be applied for aircraft HEP powertrains [5]. The feasibility of these HEP powertrains and their potential for improving the fuel- and energy efficiency of aircraft depends on the choice of HEP system architectures and power management strategy. This paper focuses on the power management and system sizing of a so-called parallel HEP architecture. This parallel architecture applies an electric propulsion powertrain in parallel to a conventional gas turbine powertrain that is typically installed as a turbofan engine. In such a parallel HEP architecture the electric powertrain supports the aircraft propulsion in flight phases where power demand is very high, typically the take off and climb phases. In addition, the electric systems in such HEP architecture can be combined well with more electric non-propulsive systems as found in MEA, yielding potential savings in overall fuel and energy consumption due to synergies in systems deployment. This paper presents an aircraft level model study of the fuel- and energysaving potential of HEP in combination with MEA retro-fitted on an A320NEO aircraft. The following main electric systems are considered: electric motors, batteries and power electronics for parallel HEP, electric components for replacement of the main hydraulic and pneumatic non-propulsive systems like the environmental control system (ECS), flight control system (FCS), ice protection system (IPS) and landing gear (LG) actuation. Besides these electric system models, also models of the aircraft, turbofan engine and flight mission are used to quantify the power and fuel needs and account for system mass changes involved with the electric components replacements. The results in terms of fuel- and energy consumption for the considered missions are reported.

\section{Modelling approach \& implementation}

\subsection{HEP architecture}

The investigations in this study focus on a parallel HEP architecture implemented as an electrically assisted turbofan powertrain. This powertrain is assumed as a traditional turbofan engine, with an electric motor that provides additional power to the Low Pressure Turbine (LPT) shaft [5].

The sizing of the electric motor and the other electric components (batteries, etc) is based on the level of power (and energy) that shall be supplied to the fan shaft. This power level is expressed as an electrification ratio $\left(\varphi_{H}\right)$ of the HEP system which is defined as the momentary electric power supply divided by the momentary total power. For simplification, the electrification ratio is assumed constant within one flight-phase. Suitable values for the electrification ratio have been determined in previous studies [6] from multiple sweeps of mission-evaluations aimed for minimal trip-fuel and -energy. The values 0.15 in take-off, 0.1 in climb and 0.0 in all other flight-phases are used in this study.

The implementation of the HEP architecture is achieved as an integrated system model in MATLAB. It integrates the other component models that will be explained in the following sub-sections.

\subsection{Engine model}


The turbofan engine is modelled with NLR's Gas-Turbine Simulation Program (GSP) [7], which is based on thermodynamic modelling of mass- and energy-balances of the main engine components (compressors, combustor, turbines, fan etc.). The GSP model allows to simulate effects on fuel consumption of the common primary aircraft operational parameters like thrust, speed, altitude and payload. But also does it allow to simulate effects on fuel consumption of secondary parameters like bleed off-takes from the various compressor stages or mechanical shaft power off-takes from the LPT or High Pressure Turbine (HPT) shafts. Vice versa, the GSP model also allows to simulate the supply of mechanical power to the LPT or HPT shafts, and the effects of that on fuel flow in the engine. That is exactly what is of interest in parallel HEP system studies: the effects on the turbofan fuel flow of mechanic power supply through electric motor drives to the LPT or HPT shafts. Moreover, for MEA system studies there is typically an interest in the effects of bleed off-take variations on fuel flow. Also these effects can be simulated with the GSP model. The use of such a GSP engine model in HEP studies is described in detail in [8].

In the present study an engine model of the CFM-LEAP-1a26, which is one of the engine options on the A320NEO aircraft, has been implemented in the GSP software. The main specifications of the CFM-LEAP engine as incorporated in the GSP model are given in the EASA type-certificate data sheet [9]. A surrogate model was derived from the GSP model for integration with the system model in MATLAB. Details on the surrogate modelling process are given in [10].

\subsection{HEP electric components models}

The electric components in the HEP system that are considered in this study are the electric motors that drive the LPT shafts, power electronics (mainly inverters), electric power cables and batteries. The electric components are included as mass contributions in the overall system model. As such, the electric components are included as basic "black box" models, with electric power and/or energy demands as inputs, and predicted component mass as output.

For electric motors and power electronics the mass is determined from the required maximum power level of the electric system and from the specific power and the energetic efficiency of these components. For batteries the mass is determined from the required maximum energy consumption of the electric system and from the specific energy of the batteries. Of course, also the required maximum power level and the specific power of the batteries is important, but in this study the maximum energy consumption of the battery dominates the sizing process. Furthermore the battery energy efficiency and minimum state of charge (SoC) are taken into account in the sizing process. The batteries' energetic efficiency accounts for the recharge energy losses and therefore is only used for the total energy calculation, not for the battery mass calculation. Energetic efficiencies of electric power cables are also accounted for. The electric components models are described in more detail in [6].

There is a strong technology development ongoing in this field of electric components, mainly driven from other industrial sectors like automotive and consumer electronics. Because this development is expected to continue in the coming decades, short term and long term levels of technology development are considered here. These levels correspond to the year 2020 onwards (expressed here as 2020+) and the year 2040 onwards (expressed here as 2040+), respectively. For both levels, performance numbers in this study were derived by averaging results from publicly available feasibility studies, as given in table 1 below. It should be noted that the uncertainty of these numbers is high because of the large spread in the numbers obtained from literature. 
Table 1. Electric components performance numbers for the two technology levels (2020+, 2040+).

\begin{tabular}{|l|r|r|}
\hline Electric components performance & $2020+$ & $2040+$ \\
\hline Batteries & & \\
\hline Efficiency [\%] & 92.5 & 95.0 \\
\hline Specific power [kW/kg] & 3.0 & 6.0 \\
\hline Specific energy [kWh/kg] & & 1.0 \\
\hline Electric motor & 95.0 & 98.0 \\
\hline Efficiency [\%] & 7.5 & 15.0 \\
\hline Specific power [kW/kg] & & \\
\hline Power electronics & 95.0 & 98.0 \\
\hline Efficiency [\%] & 7.5 & 15.0 \\
\hline Specific power [kW/kg] & & \\
\hline Power cables & 99.0 & 99.6 \\
\hline Efficiency [\%] & &
\end{tabular}

With these electric components performance numbers, the sizing of the components and their weight calculations can be done from the power and energy requirements that come from the aircraft level analysis. Of course, these component weights and sizes have effects on the aircraft level power and energy evaluations and therefore shall be determined in an iterative system level design process. This process is explained in [10].

\subsection{Aircraft model}

For the prediction of the aircraft thrust requirement during the mission a basic aerodynamic "point mass" model is used in this study. Only forward flight and flight path angle is included in the present study's flight mission; turns and manoeuvres and roll and yaw rotations are not considered.

The main characteristics like the various mass components (airframe, engines, electric components, fuel, payload) and the aircraft lift and drag coefficients are included in this model. Because the HEP system investigations are focussed on certain flight phases, the aircraft model is intended to be representative for these flight phases, including taxi-out, taxi-in, take-off, climb and descent/landing. To account for this, also the dependency of the aerodynamic coefficients on flap and gear settings and Mach number and the ground rolling friction are incorporated, as well as the actual time-dependent fuel mass. Hence the aircraft model also allows for simplified calculation of the required thrust during taxi, take-off, descent and landing. This model is described in more detail in [10].

The implementation of this aircraft model has been achieved in MATLAB. The main model parameters used for the A320NEO aircraft (A320-251N, [11],[12]) that is considered in this study are as follows: Max. take-off mass (mTOM) 73.5t (tonnes), Operating Empty mass (mOE) 45.7t, Max. landing mass (mLM) 66.3t, wing area $122.0 \mathrm{~m}^{2}$.

\subsection{Mission model}

The mission model produces all the relevant flight path variables (distance, altitude, speed, flight path angle, flap and landing gear settings) as a continuous function of time. As inputs, these variables shall be prescribed at the start and end of each of the considered flightphases expressed as function of distance (Table 2). Linear interpolation between start and end of each flight-phases is used for each of the prescribed variables. Flight time for each flight-phase is calculated from the distance travelled at the interpolated speed.

Table 2. Inputs for the relevant flight path variables that are used in the mission model, values derived from (Airbus, 2002). Flight phase indicators: (0): start of mission; (1): end of taxi out; (2) end of take-off; (3) end of climb; (4) end of cruise; (5) end of descend; (6) end of taxi in. Also bleed air 
mass flow fractions and LPT shaft power off-takes, per engine for the reference aircraft, and nonpropulsive electric power demands for the MEA architecture are given.

\begin{tabular}{|l|r|r|r|r|r|r|r|}
\hline Flight-phase id & \multicolumn{1}{c}{$(\mathbf{0})$} & $(1)$ & \multicolumn{1}{c}{$(3)$} & $(4)$ & \multicolumn{1}{c}{$(5)$} & \multicolumn{1}{c}{$(6)$} \\
\hline Distance [km] & 0 & 4.5 & 18 & 289 & 1308 & 1514 & 1534 \\
\hline Altitude [m] & 0 & 0 & 457 & 11277 & 11277 & 457 & 0 \\
\hline Calibrated air speed [m/s] & 0 & 15 & 129 & 130 & 130 & 129 & 15 \\
\hline flight path angle [deg] & 0 & 0 & 5 & 1 & 0 & -3 & 0 \\
\hline flap settings [deg] & 0 & 42 & 0 & 0 & 0 & 35 & 0 \\
\hline landing gear settings [-] & 1 & 1 & 0 & 0 & 0 & 1 & 1 \\
\hline electrification ratio [-] & 0 & 0 & 0.15 & 0.1 & 0 & 0 & 0 \\
\hline bleed air mass flow [\%] & 0 & 0.1 & 0.03 & 0.05 & 0.06 & 0.1 & 0.04 \\
\hline LPT-offtake [kW] & 0 & 35 & 37 & 42 & 40 & 35 & 35 \\
\hline MEA power demand [kW] & 0 & 258 & 256 & 352 & 354 & 286 & 204 \\
\hline
\end{tabular}

\subsection{System model}

All the models described in the previous sub-sections are integrated in an aircraft level system model. This system model is implemented in MATLAB and is used in the aircraft level design process for the iterative evaluation of power and energy consumption during the considered mission. A schematic representation of this iterative evaluation process with the system model is given in (Fig. 1) below.

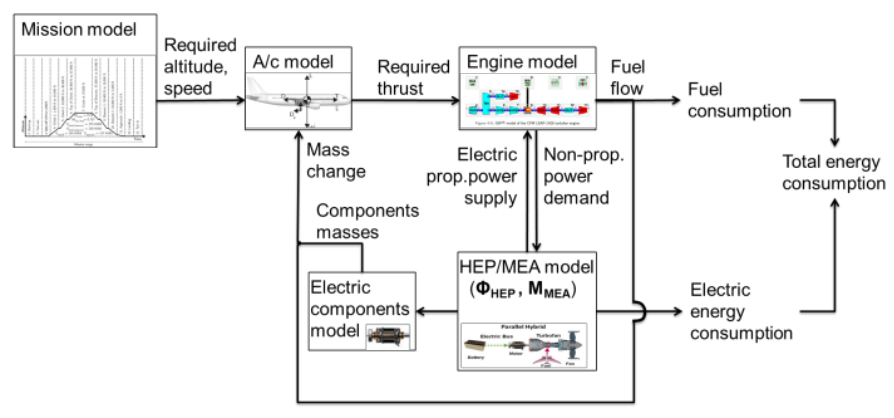

Fig. 1. Schematic of the iterative system level design process with the system model for the aircraft level power and energy evaluation.

In the present study, the mission evaluation is first performed for the reference aircraft, i.e. the A320NEO with the LEAP-1A engine without any HEP or MEA systems. Then subsequently the various components of the HEP and MEA systems are included in the system model and the corresponding power demands and systems sizing are accounted for. First the evaluations will be done using the performance numbers of the electric components for the $2020+$ technology level and subsequently for the $2040+$ technology level. This procedure will be briefly explained in the following section, along with the presentation of the results.

\section{RESULTS}

For the HEP and MEA system sizing we consider as mission requirements, among others, a short mission range of $1500 \mathrm{~km}(800 \mathrm{~nm})$, cruise speed of Mach 0.78 , and a payload of 150pax (i.e. 14.3t). Furthermore a fixed fuel mass of $1.8 \mathrm{t}$ is used, which is assumed as the total of alternate, contingency and reserve fuel masses (estimated from [13]). Also the system sizing respects the main aircraft level specifications of the A320NEO like mTOM. 


\subsection{Reference aircraft}

In the reference aircraft mission evaluation the fuel consumption is calculated with the system model presented in the previous section. This mission evaluation includes representative settings for bleed air off-takes and mechanical shaft-offtakes from the engines. The reference aircraft model is based on the A320NEO and does not comprise any of the HEP or MEA components. The bleed air mass flow fractions and LPT shaft power off-takes in all flight phases that are used are given in the table 2.

From the reference aircraft mission evaluation it is found that the trip fuel consumption for this mission is about 5.5t. This results in a take-off mass for the reference aircraft of 67t. Because 73.5t is used as the mTOM of the A320-NEO [9], this leaves an approximate 'mass budget' of about $6.5 \mathrm{t}$ that can be spent on HEP and MEA system components.

\subsection{HEP}

First we consider the sizing of only the HEP system for the parallel hybrid architecture as explained above (sec. 2.1). The main change in comparison to the reference aircraft is the installation of an electric motor on the fan shaft of the turbofan, batteries for the electric energy supply of electric motor and the necessary wiring and power electronics. The turbofan engine is not changed. Effectively in the system model these changes are included as additional system masses from all the electric equipment and as mechanical power supply (from the electric motor) to the fan shaft of the engine model. The sizing of the electric components is based on the level of power that shall be supplied to the fan shaft, which depends on the total propulsive power demand and the electrification ratio of the HEP $\left(\varphi_{H}\right)$ (see table 2). The sizing of the electric equipment for the considered mission and for the $2020+$ technology level yields a total mass increase of about $5906 \mathrm{~kg}$, resulting in higher lift-, drag- and required thrust forces. Integration of the fuel flow over the whole mission, as calculated with the engine model, leads to an overall change in trip fuel of about $+2 \%(126 \mathrm{~kg})$ and trip energy of about $+5 \%$, in comparison with the reference aircraft.

\subsection{HEP + 90\%TF}

The electric components of the HEP system introduce additional mass, which obviously leads to increased fuel consumption $(+2 \%)$. But the HEP also provides additional power to the fan shaft, which allows to reduce the maximum power capacity of the turbofan (TF) core. Therefore we also include the downscaling of the TF in the engine model, here approximated as a reduction of engine core diameter with corresponding mass reduction and other dependent engine parameters like mass flow and shaft speed as explained in [6]. In this way the GSP engine model is extended and allows to predict the main effects in engine mass and fuel flow for downscaled engine size. With the $15 \%$ power supply from the HEP system in take-off condition it was estimated that $15 \%$ downscaling of the TF engine would be allowable. However it turned out from the mission evaluations with the extended GSP engine model that in this case more than 10\% downscaling (i.e. below $90 \%$ of the reference core diameter) yields in-allowable exhaust gas temperatures at the HPT first stage. Therefore we limit the TF engine downscaling to $90 \%$, expressed here as $90 \% \mathrm{TF}$. This results in, among others, an engine mass reduction of about $400 \mathrm{~kg}$ (total for two engines) and slightly improved cruise SFC (SFC decreases with 3\%: from $17.7 \mathrm{~g} / \mathrm{kN} / \mathrm{s}$ to $17.1 \mathrm{~g} / \mathrm{kN} / \mathrm{s})$.). This yields an overall change in trip fuel of about $-5 \%(273 \mathrm{~kg})$ and trip energy of about $-5 \%(-3 \mathrm{MWh})$, in comparison with the HEP-only aircraft. 


\subsection{HEP + 90\%TF + MEA}

To better exploit the electric systems of the HEP architecture and to optimize synergies in systems deployment, we also include more electric non-propulsive systems. In such a MEA system architecture, all pneumatic and hydraulic components in the non-propulsive systems, like ECS, ice protection, landing gear, flight controls, are replaced by electric systems. This was investigated in some detail for single aisle aircraft in [15], and the main changes in system masses are given in table 3 below.

Table 3. Mass changes for converting from conventional to electrical subsystem architecture [15].

\begin{tabular}{|l|l|}
\hline MEA system & Mass change [kg] \\
\hline Actuation of FCS and LG & -455 \\
\hline LG brakes and steering & -99 \\
\hline IPS & +34 \\
\hline ECS & +80 \\
\hline Other (removal of hydraulic and pneumatic systems) & -540 \\
\hline \hline Total & $\mathbf{- 9 8 0}$ \\
\hline
\end{tabular}

The non-propulsive power demands for the MEA architecture during the mission are assumed to be constant per flight phase [15], [14], as listed in Table 2. For this configuration, the sizing of the electric equipment yields a total mass increase of about 359 $\mathrm{kg}$ (1339 kg increase due to additional battery mass minus $980 \mathrm{~kg}$ saving due to application of the MEA systems architecture).). Integration over the whole mission of the fuel flow leads to an overall change in trip fuel of about $-7 \%(\sim 380 \mathrm{~kg})$ and trip energy of about $-7 \%$, in comparison with the HEP $+90 \% \mathrm{TF}$ aircraft. To clarify which mission and which aircraft architecture are considered in this section, we use the colouring scheme: blue for the reference aircraft and red, green, purple for the various HEP architectures. An overview of the main results from the mission evaluations for the HEP architectures is given in Fig. 2.

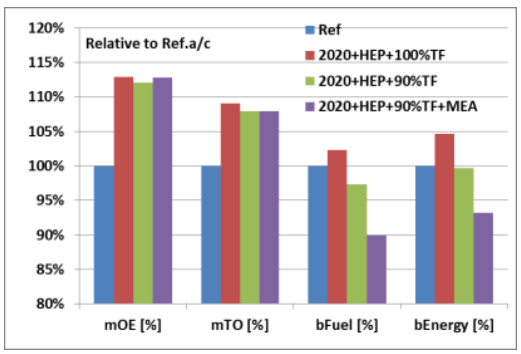

Fig. 2. Main masses (operative empty mass (mOE) and take-off mass (mTO)) and performance results (trip fuel mass (bFuel) and trip energy (bEnergy)) of the analysed HEP architectures according to the $2020+$ scenario. All results are given as relative change in percent compared to the values that were found for the reference aircraft, and shown in the colouring scheme for the HEP architectures.

\section{Conclusions}

An aircraft level model study has been executed of the fuel- and energy-saving potential of HEP in combination with MEA retro-fitted on an A320NEO aircraft. System models of the aircraft, turbofan engine, the more electric components and the flight mission are used to quantify the power and fuel needs and account for system mass changes involved with the electric components replacements. The results in terms of fuel- and energy consumption for the considered missions, as well as some transport energy metrics are reported.

The aim with the considered parallel HEP architecture is to electrically support the turbofan engine in the flight phases where thrust demand is high and efficiency is low, thus 
allowing for down-scaled, smaller, lighter and more efficient turbofan engine core. In combination with MEA the potential efficiency can be further improved.

A potential trip fuel reduction of about $10 \%$ is found for the HEP $+90 \% \mathrm{TF}+\mathrm{MEA}$ architecture with 2020-level of electric technologies. About half of this reduction comes from the HEP with downscaled engine (HEP+90\%TF) and the other half from the introduction of the MEA systems. But it should be noted that this reduction is found in this study where only short mission range $(800 \mathrm{~nm})$ is considered.

Acknowledgement: This work has received funding from the Clean Sky 2 Joint Undertaking under the European Union's Horizon 2020 research and innovation program under grant agreement CS2-LPA-GAM-2018/2019-01 (NOVAIR).

\section{References}

1. Airbus, Global market forecast 2018-2037. (2018)

2. Flightpath 2050 Europe's Vision for Aviation - European Commission

3. ACARE - Advisory Council for Aviation Research and innovation in Europe, ACARE Strategic Research and Innovation Agenda (SRIA), 2017

4. C. Perullo, D. Mavris. A review of hybrid-electric energy management and its inclusion in vehicle sizing. Aircr. Eng. Aerosp. Technol. 86: 550-557, 2014.

5. J.L. Felder, NASA Glenn Research Center, "NASA Hybrid Electric Propulsion Systems Structures," presentation to the committee on September 1, 2015.

6. S.C. Tan, Electrically Assisted Propulsion \& Power Systems for Short Range Missions: Electrification of a Conventional Airbus A320neo, MSc. Thesis TU Delft, 2018, NLRTR-2018-153.

7. Team GSP 11 User Manual Version 11.4.5.0. Netherlands Aerospace Centre (NLR) https://www.gspteam.com/index.html, 2015.

8. A.W.X. Ang, A.G. Rao, T. Kanakis, W. Lammen, Performance analysis of an electrically assisted propulsion system for a short range civil aircraft $\mathrm{J}$ Aerospace Engineering, 233(4), 1490-1502, 2018.

9. European Aviation Safety Agency (EASA), Type-Certificate Data Sheet: No. E .110 for Engine LEAP-1A \& LEAP-1 C series engines, 2018.

10. W.L. Lammen, W.J. Vankan, Electrification studies of single aisle aircraft: a 'retrofit' investigation including parallel hybrid electric propulsion, International Symposium on Sustainable Aviation, Budapest, Hungary, 26 - 29 May 2019.

11. Airbus, https://www.airbus.com/aircraft/passenger-aircraft/a320-family/a320neo.html (accessed 14-05-2019)

12. Airbus Customer Services, Getting to Grips with Aircraft Performance, 2002.

13. International Civil Aviation Organization (ICAO), Annex 6 Operation of Aircraft Part 1: International Commercial Air Transport - Aeroplanes, 2010.

14. D. Scholz, R. Seresinhe, I. Staack, C. Lawson, 2013, Fuel Consumption due to Shaft Power Off-Takes from the Engine, 4th International Workshop on Aircraft System Technologies, AST 2013.

15. I. Chakraborty, D. N. Mavris, M. Emeneth, A. Schneegans, “An Integrated Approach to Vehicle and Subsystem Sizing and Analysis for Novel Subsystem Architectures," in Proceedings of the Institution of Mechanical Engineers, Part G: Journal of Aerospace Engineering, vol. 230, pp. 496-514, 2016. 